\title{
Anti-ulcer Agent
}

National Cancer Institute

\section{Source}

National Cancer Institute. Anti-ulcer Agent. NCI Thesaurus. Code C29701.

Natural or synthetic Anti-ulcer Agents relieve and reduce the symptoms of ulcers in the stomach and upper small intestine, systemically and locally, by reducing gastric secretion (proton pump inhibitors), neutralizing hyperacidity (alkalinizing agents), or improving healing and protecting the mucosa (sucralfate). Recurrent gastric and duodenal ulcers caused by Helicobacter pylori infections are treated with antibiotic therapy. 